\title{
Choice discrimination and fixed-interval performance
}

\author{
DONALD MELTZER, KRISTIN ROBERTSON, and JOHN IRWIN \\ Southern Illinois University, Carbondale, Illinois
}

\begin{abstract}
Four rats were reinforced on a mixed fixed-interval, fixed-interval (FI,FI) schedule. The short FI was $15 \mathrm{sec}$, and the long FI was $60 \mathrm{sec}$. Two levers were available to the subject. Responses on one were reinforced following short intervals, and responses on the other were reinforced following long intervals. All subjects learned the temporal discrimination. They responded at higher rates on the short lever early in the interval and on the long lever late in the interval. Duration of the preceding interval had no effect on the distribution of responses, but subjects made slightly more responses following long intervals.
\end{abstract}

Fixed-interval (FI) schedules have been used to study many aspects of animals' temporal processes. One issue that has been examined is the effect of prior events on the pattern and rate of a subject's responses during the current interval. Omission or reduction of reinforcement at the end of a preceding interval has been shown to increase response rate during the next interval (see, e.g., Meltzer \& Howerton, 1973; Staddon \& Innis, 1969). Duration of the preceding interval also affects response rate in the next interval. Innis (1978) showed that when two different interval durations were used in the same session, longer prior interval durations increased the response rate of pigeons during the current interval. Meltzer (1987) found the same effect of prior interval duration when rats were the subjects but also showed that preceding interval duration did not affect response distribution within the current inter$\mathrm{val}$. In other words, the proportion of total responses that a subject had emitted one third and two thirds of the way through an interval was approximately the same following long and short intervals. These data suggested that preceding interval duration affected performance rather than temporal discrimination.

Another question that has been examined is whether increased rates following longer intervals are caused by the fact that subjects have responded over the course of the longer interval or whether it is a consequence of the longer time between reinforcements at the beginning and end of the long interval. For instance, a subject responding at the beginning of the current interval would receive two reinforcements in the last $30 \mathrm{sec}$ if the preceding interval was an FI of $30 \mathrm{sec}$, but would receive two reinforcements over the last $2 \mathrm{~min}$ if the preceding interval was an FI of $2 \mathrm{~min}$. Meltzer and Robertson (1990) reinforced rats on an FI 2min schedule but restricted the portion of the interval during which the lever was available to the subject. It was in-

\footnotetext{
Reprints may be requested from Donald Meltzer, Department of Psy-
} chology, Southern Illinois University, Carbondale, IL 62901-6502. serted for either the last $40 \mathrm{sec}$ or the last $80 \mathrm{sec}$ of the interval, with each type of interval occurring equally often. Thus, the time during which subjects could respond varied, but the time between reinforcements was fixed at $2 \mathrm{~min}$. The sequence of intervals was arranged so that there was an equal probability of long and short lever availability in the current interval, regardless of whether the lever had been available for a long or a short time in the preceding interval. The rats responded at higher rates during both types of current interval when the lever had been available for only $\mathbf{4 0} \mathrm{sec}$ during the preceding interval. These data suggest that earlier results showing increased response rates when the preceding interval was longer may have been a consequence of the longer delay of reinforcement rather than the longer time over which the animal could respond.

In the present study, the effect of prior interval duration on FI performance was examined in a different way. Subjects were reinforced at the end of short intervals for responses on one lever and at the end of long intervals for responses on another. However, there were no discriminative stimuli associated with interval duration. A similar procedure, in which pigeons served as subjects, was used by Catania and Reynolds (1968). There were two different fixed intervals in each session, only one of which was in effect at any given time. The same number of long and short intervals occurred during each session. Since only one key was available to the subject, the distribution of responses during long intervals was bimodal, with a peak in response rate at approximately the time when the short interval would have ended and a second peak at the end of the long interval. The first peak presumably reflected control by the short interval, which caused responses to increase until that time and then caused them to gradually decrease. Unfortunately, the authors did not report whether the sequence of intervals was controlled, so that long intervals were equally probable following long and short intervals, and short were also equally probable. Nor did they examine the data for any effects of preceding interval duration. 
Two questions were considered in the present study. One was whether animals would shift from the short lever to the long lever as a function of time since reinforcement. Since there have been repeated demonstrations that animals can discriminate between stimuli of different durations by choosing different response alternatives (see, e.g., Church \& Deluty, 1977; Stubbs, 1968), as well as evidence of temporal discrimination in the Catania and Reynolds (1968) study, there was good reason to expect that rats would shift from the short to the long lever during the course of the long FI. The second question was whether preceding interval duration would affect total response output, the distribution of responses on the two levers, or both.

\section{METHOD}

\section{Subjects}

The subjects were 4 male Long-Evans rats 120-140 days old at the beginning of the experiment. Each animal was maintained at $85 \%$ of its free-feeding weight by food reinforcement and supplementary feeding. Water was always available in the animals' home cages.

\section{Apparatus}

Three identical experimental chambers with interior dimensions of $21.0 \times 30.5 \times 18.0 \mathrm{~cm}$ were used. Two BRS/LVE retractable rodent levers were mounted $3.5 \mathrm{~cm}$ above the floor and $4 \mathrm{~cm}$ from the side walls. The levers required a minimum force of approximately $0.2 \mathrm{~N}$ for operation. Lever insertion and retraction both took approximately $2 \mathrm{sec}$. A pellet dispenser delivered $45-\mathrm{mg}$ food pellets to a hopper mounted at the midline of the front wall, just above the floor. Two incandescent white lights, each emitting approximately $127 \mathrm{~cd} / \mathrm{m}^{2}$ measured $0.5 \mathrm{~cm}$ from the source, were located on the front wall. One light was centered $4.2 \mathrm{~cm}$ above the lever (i.e., $7.7 \mathrm{~cm}$ above the floor and $4.0 \mathrm{~cm}$ from the left wall). The other light was at the same height and $4.0 \mathrm{~cm}$ from the right wall. Both lights remained on throughout an experimental session. An Apple Ile computer and solid-state interface equipment, which were located in an adjacent room, provided experimental control and recording.

\section{Procedure}

The subjects were trained to eat from the magazine during the first session. Autoshaping began in Session 2. Both levers were retracted when subjects were placed in the experimental chamber. After $30 \mathrm{sec}$, both levers were inserted and the lights were turned on. If the rat pressed either lever, a food pellet was delivered, the lights were turned off, and the levers were retracted. If the rat failed to respond, a food pellet was delivered at the end of $8 \mathrm{sec}$, the lights were turned off, and the levers were retracted. After the 30-sec intertrial interval, the levers were inserted again and the procedure was repeated until the rat had received 30 reinforcements. Since all the rats developed a high probability of responding on one lever after two or three sessions, only the less preferred lever was introduced during the following one or two sessions. There were two additional sessions in which the left and right levers were inserted in a semirandom sequence while all the other parameters remained unchanged.

\section{S11}

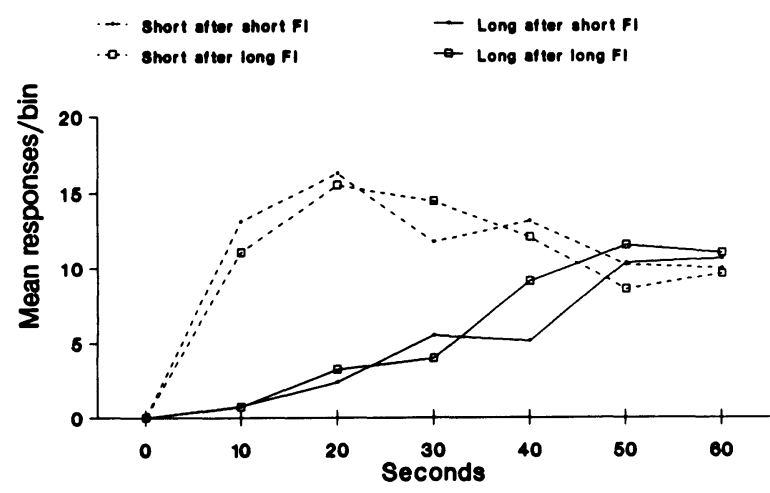

S15

- Short after short FI

a. Short after long FI

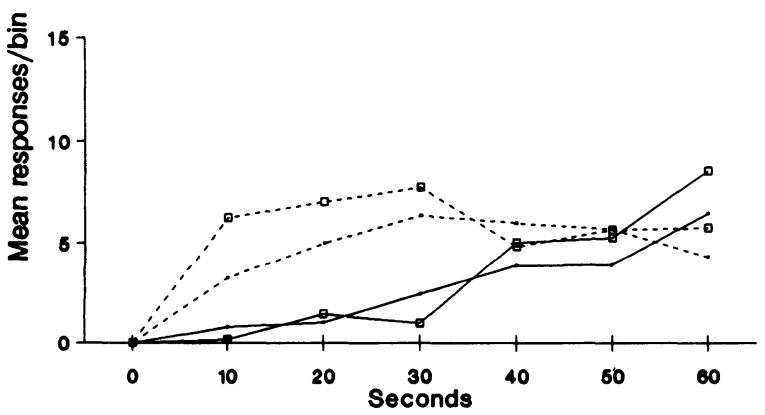

$S 13$

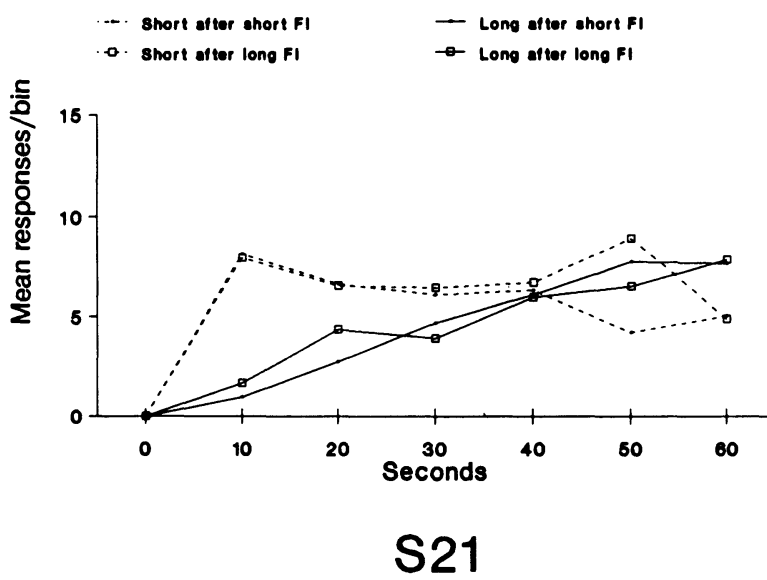

... Short after short FI

o. Short after long FI

- Long after short F

$\rightarrow$ Long after long FI

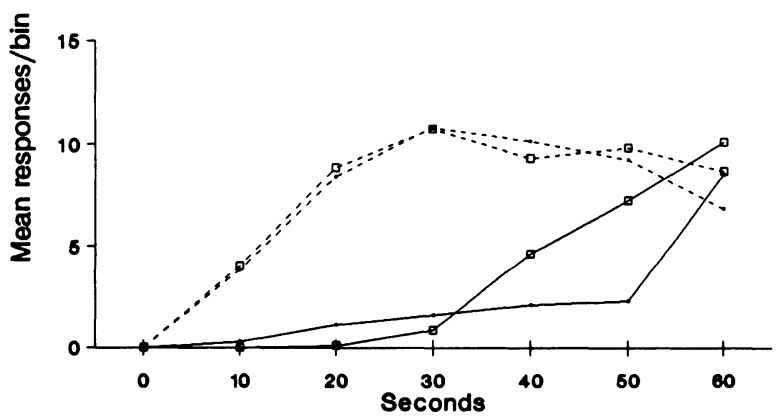

Figure 1. The mean number of responses on the short and long levers during each 10-sec bin of the 60-sec interval are shown above. Responses on the short lever were recorded separately following short FIs (short lever after short FI) and long FIs (short lever after long FI) as were responses on the long lever. 
Table 1

The Mean Long and Short Lever Responses during a 60-Sec Interval, with Total Responses on Both Levers

\begin{tabular}{clrrr}
\hline & & \multicolumn{3}{c}{ Preceding Interval } \\
\cline { 3 - 5 } Subject & Lever & \multicolumn{1}{c}{ All } & Short & Long \\
\hline S11 & Short & 18.20 & 18.61 & 17.80 \\
& Long & 9.29 & 8.68 & 9.90 \\
& Both & 27.49 & 27.29 & 27.69 \\
S13 & Short & 9.63 & 9.07 & 10.20 \\
& Long & 7.48 & 7.43 & 7.52 \\
& Both & 17.11 & 16.50 & 17.72 \\
S15 & Short & 8.39 & 7.56 & 9.23 \\
& Long & 4.92 & 4.55 & 5.29 \\
& Both & 13.32 & 12.11 & 14.52 \\
S21 & Short & 12.87 & 12.58 & 13.17 \\
& Long & 4.89 & 4.00 & 5.78 \\
& Both & 17.76 & 16.58 & 18.95 \\
\hline
\end{tabular}

Following autoshaping, the rats were placed on a mixed FI, Fl schedule. During these sessions, intervals were either 15 or $60 \mathrm{sec}$ long. One lever was arbitrarily assigned as the short lever (the left for 2 subjects and the right for the other 2). The other lever was the long lever.

The 15-sec interval was followed by a 3-sec interval, at the end of which a response on the short lever was reinforced. However, responses on the long lever at any time during the 3-sec interval reset the 3-sec clock. A subject could remain in this segment of the schedule indefinitely by pressing the incorrect lever repeatedly. A similar 3-sec interval followed the 60-sec interval, except that long responses were reinforced and short responses reset the clock. The first reinforcement followed a response on the short lever in half the sessions and the long lever in the remaining sessions. There were no stimulus changes as a consequence of an incorrect response. Both levers were retracted for $10 \mathrm{sec}$ following each reinforcement. Lever retraction and insertion each took $2 \mathrm{sec}$.

Interval sequences were arranged so that a 15 -sec interval was equally likely to follow a 15 - or 60 -sec interval. A $60-\mathrm{sec}$ interval also was equally likely to be followed by a $15-$ or $60-\mathrm{sec}$ interval. Thus there was no possibility that a subject would learn to respond at higher or lower rates during an interval, because there was a differential probability that it would be followed by a long or short interval. The numbers of short and long responses were recorded in six successive 10-sec bins during the 60-sec intervals, except during the 60-sec interval that began half the sessions. Responses were recorded separately during intervals that followed FI 15-sec and FI 60-sec intervals. The procedure was followed for 28 sessions, and subjects earned 17 reinforcements in each session.

\section{RESULTS}

Data from the last eight sessions of the experiment were analyzed. The actual mean time to reinforcement following lever insertions in short intervals ranged from $20.2 \mathrm{sec}$ for subject S11 to $21.4 \mathrm{sec}$ for $S 13$. The actual mean time to reinforcement in long intervals ranged from $65.8 \mathrm{sec}$ for S11 to $67.1 \mathrm{sec}$ for S15. Subjects actually made a sub-

\section{S11}

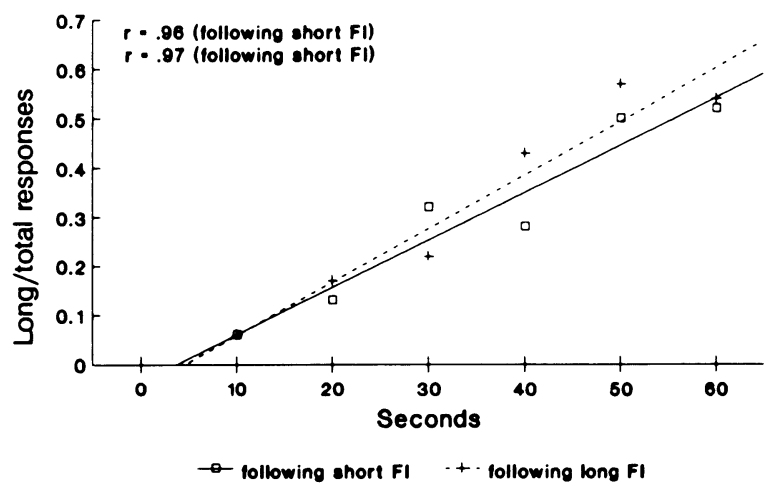

S15

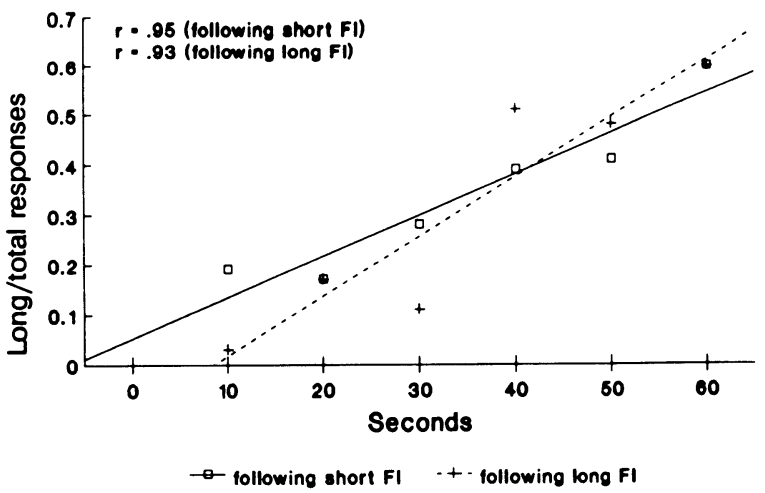

S13

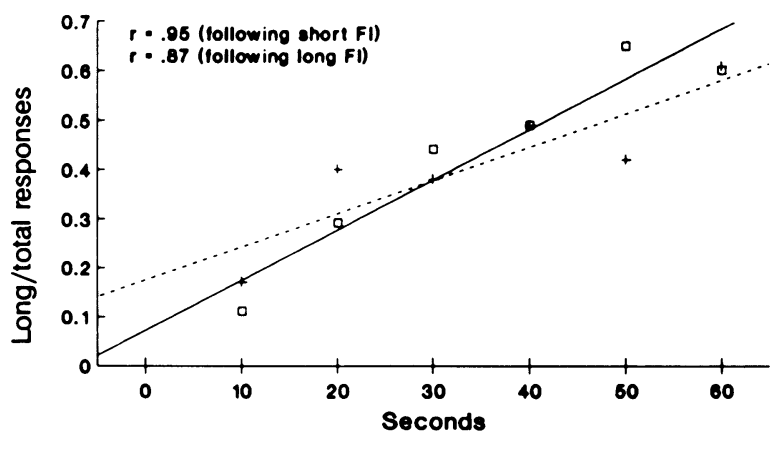

$\rightarrow$ following ahorl FI + + following long FI

S21

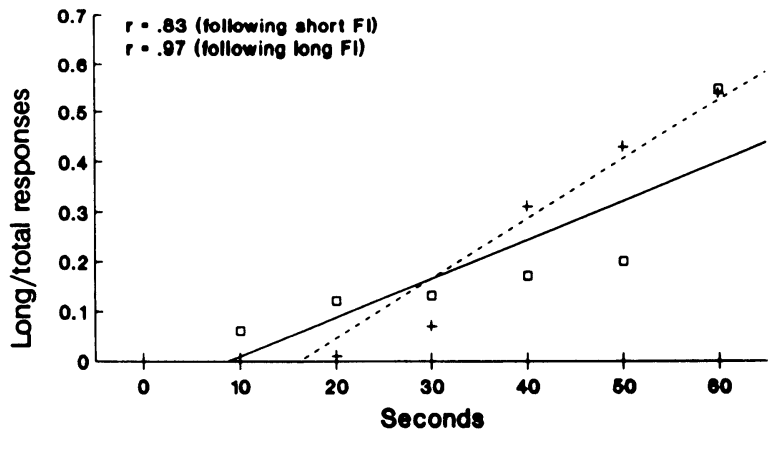

Figure 2. The ratio of responses on the long lever to total responses in successive 10-sec bins during the 60-sec interval are shown above. Data from intervals following short and long intervals were separated, and linear regression lines were fitted to each data set. The product-moment correlation coefficients $(r)$ are included and show ratio as a function of time. 
stantial number of incorrect responses during the 3-sec correction intervals, but most incorrect responses occurred in bursts. During the last eight sessions, there were no incorrect responses in more than half of the 3-sec intervals and large numbers of incorrect responses in a few intervals. Computer limitations made it impossible to analyze the data more precisely.

Figure 1 shows the mean numbers of long and short lever responses in successive 10-sec bins during the eight sessions. Responses on the long lever increased throughout the interval. Responses on the short lever either decreased, or first increased and then decreased. All subjects responded at higher rates on the short lever during the first $40 \mathrm{sec}$ of the interval and at higher rates on the long lever during the final $10 \mathrm{sec}$ of the interval. However, it was apparent that the rate of increase in responding on the long lever was greater, and in most cases more regular, than the rate of decrease on the short lever. Figure 1 also shows that the effect of preceding interval duration was small and inconsistent both between and within subjects. However, all subjects made more total responses during intervals that followed long rather than short intervals. Table 1 shows that the differences generally were small and did not have a consistent effect on short and long lever responses.

Figure 2 shows the ratio of long lever responses to total responses during each $10-\mathrm{sec}$ bin. This ratio is a derived measure of temporal discrimination. Responses following short intervals were separated from those following long intervals. A linear function that was fit to the data accounted for at least $80 \%$ of the variance for all subjects. Although the slope of the regression line was steeper for 3 of the 4 subjects following long intervals, the differences were small, and there was no consistent difference in the proportion of variance accounted for by preceding interval duration.

\section{DISCUSSION}

All subjects clearly learned to shift their response distribution from the short to the long lever as the interval progressed. Since it has long been assumed that fixed-interval performance was based on temporal discrimination, these results were not surprising. Nevertheless, the opportunity to modify response choices as the interval progressed represented a combination of the standard fixed-interval procedure and other studies of temporal discrimination of stimulus duration. Gibbon and Allan (1984) have provided an extensive review of the methods and results of these studies. One of the most common techniques involves the presentation of either a short or a long stimulus, after each of which a different response is reinforced (see, e.g., Church \& Deluty, 1977; Stubbs, 1968). The results showed that animals discriminated between stimuli of different durations and were equally likely to make "long" and "short" responses when presented with a stimulus of a duration equal to the geometric mean of the two training stimuli. In the present study, responses on the long lever did not exceed responses on the short lever until long after the geometric mean of the two intervals. Any number of variables may account for the difference; among them might be the use of discrete trials as opposed to free operants, the fact that subjects always earned a reinforcement at the end of the interval in this study as compared to nonreinforcement following an incorrect response in the discrete trial procedure, and the difference between monitoring an external stimulus as opposed to the passage of time during which the subject itself is responding.

Another interesting result was the much larger number of responses on the short, as opposed to the long, lever. When responses on the short lever alone were considered, one might have expected results similar to those of experiments (e.g. , those of Catania, 1970; Meck \& Church, 1984) in which animals were given discrete trials, some of which ended with reinforcement for a response after a given period of time had elapsed. No reinforcement was delivered on the remaining trials, and these trials were of much longer duration than the reinforced trials. Animals developed a pattern of responding on these nonreinforced trials in which response rate increased until approximately that point at which reinforcement was sometimes delivered and then gradually declined. Responses on the short lever declined much more slowly in this experiment. Again, several different variables may have been responsible for the difference in results. One obvious possibility is that the same reinforcement was delivered at the end of long and short intervals in this study and may have served to maintain responding on the short lever.

Finally, the ratio of long lever to total responses as the interval progressed did not change as a function of whether the preceding interval was long or short. This is consistent with Meltzer's (1987) results, which show that preceding interval duration did not affect response distribution within the current interval. Preceding interval duration affects response output but does not affect temporal discrimination.

\section{REFERENCES}

Catania, A. C. (1970). Reinforcement schedules and psychophysical judgments: A study of some temporal properties of behavior. In W. N. Schoenfeld, The theory of reinforcement schedules (pp. 1-42). New York: Appleton-Century-Crofts.

Catania, A. C., \& Reynolds, G. S. (1968). A quantitative analysis of the behavior maintained by interval schedules of reinforcement. Journal of Experimental Psychology: Animal Behavior Processes, 11, 327-383.

Church, R. M., \& Deluty, M. Z. (1977). Bisection of temporal intervals. Journal of Experimental Psychology: Animal Behavior Processes, 3, 216-228.

Gibbon, J., \& AllaN, L. (Eds.) (1984). Timing and time perception (Annals of the New York Academy of Sciences, Vol. 423). New York: New York Academy of Sciences.

INNIS, N. K. (1978). Contrast effects in multiple fixed-interval reinforcement schedules. Journal of the Experimental Analysis of Behavior, 29, 233-242.

MECK, W. H., \& CHURCH, R. M. (1984). Simultaneous temporal processing. Journal of Experimental Psychology: Animal Behavior Processes, $10,1-29$.

Meltzer, D. (1987). Sequential effects of interval duration on fixedinterval performance. Journal of the Experimental Analysis of Behavior, 47, 73-80.

Meltzer, D., \& Howerton, D. L. (1973). Sequential effect of reinforcement magnitude on fixed-interval performance in rats. Journal of Comparative \& Physiological Psychology, 85, 361-366.

Meltzer, D., \& RoberTsON, K. (1990). Sequential effects of responseopportunity duration. Bulletin of the Psychonomic Society, 28, 426-428.

STADDON, J. E. R., \& INNIS, N. K. (1969). Reinforcement omission on fixed-interval schedules. Journal of the Experimental Analysis of Behavior, 12, 689-700.

STUBBS, A. (1968). The discrimination of stimulus duration by pigeons. Journal of the Experimental Analysis of Behavior, 11, 223-258.

(Manuscript received March 19, 1991.) 